\title{
Multi-spacecraft study of the interaction between an interplanetary shock and a solar wind flux rope
}

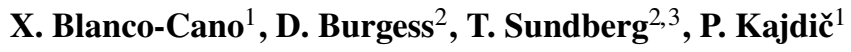 \\ ${ }^{1}$ Instituto de Geofisica, UNAM, Cd. México
${ }^{2}$ School of Physics and Astronomy, Queen Mary University of London, UK \\ ${ }^{3}$ Swedish Defence Research Agency, SE-16490, Stockholm, Sweden
}

\section{Key Points:}

- We show that the interaction of an interplanetary shock with a small flux rope can change the shock geometry affecting ion injection processes, the fluxes of energetic particles, and the upstream and downstream regions. 


\section{Abstract}

Interplanetary (IP) shocks are driven in the heliosphere by fast coronal ejecta, they can accelerate particles and are associated with solar energetic particle and energetic storm particle (ESP) events. IP shocks can interact with structures in the solar wind, and with magnetospheres. We show how the properties of an IP shock change when it interacts with a small scale flux rope like structure (FRLS). Data from CLUSTER, WIND and ACE show that the spacecraft observed the shock-FRLS interaction at different stages of evolution. WIND and ACE observed the FRLS at shock crossing, Cluster observed the FRLS downstream, after it had crossed the shock. The shock-FRLS interaction changes shock geometry, affecting ion injection processes, energetic particles fluxes, and the upstream/downstream regions. While WIND and ACE observed a quasi-perpendicular shock, CLUSTER crossed a quasi-parallel shock and a foreshock with a variety of ion distributions. The FRLS modified the shock on scales of at least $\sim 10-20 R_{E}$. The complexity of the ion foreshock measured by Cluster is explained by the dynamics of the shock transitioning from quasi-perpendicular to quasi-parallel, and the geometry of the magnetic field within the flux rope. Fluxes of particles with energy up to $125 \mathrm{keV}$ are affected by the FRLS-shock interaction, modulating the associated ESP event. The interaction of a FRLS with an IP shock has not been discussed before using multispacecraft observations. Interactions like this should occur often along the shock fronts, hence they are important for a better understanding of shock structure, evolution, and particle acceleration.

\section{Introduction}

Interplanetary (IP) shocks are large scale perturbations that propagate in the heliosphere changing the solar wind properties. In turn these shocks can be modified by the conditions that they find upstream of them and by large and small scale structures such as magnetic clouds [Burlaga et al., 1981] and small scale flux ropes [Moldwin et al., 2000]. In this work we present a multispacecraft study of the changes that an IP shock can suffer via interaction with a small scale flux rope like structure (FRLS). We find that the shock geometry and local energetic particle population can be strongly modified by this interaction.

IP shocks are very important because they play an active role in particle acceleration, being able to accelerate particles to ver high energies, i.e., tens of $\mathrm{MeV}$ (see, for example, the reviews of Lee et al. [2012] and [Reames, 2013]), and some can produce geomagnetic activity [Gonzalez et al., 1999].

IP shocks are generated in the heliosphere when a fast interplanetary coronal mass ejection (ICME) propagates in the solar wind, or at a stream interface by the interaction of fast solar wind with slow solar wind flow. The structure of the shock depends on its strength, given by the upstream magnetosonic Mach number $\left(M_{m s}\right)$ and the compression ratio $\left(B_{d} / B_{u}\right)$; on the geometry, given by $\theta_{B N}$ (the angle between the shock normal and the upstream magnetic field); and on the upstream plasma beta $(\beta)$. Shocks are classified as quasi-perpendicular (quasi-parallel) when $\theta_{B n}>45^{\circ}\left(\theta_{B N} \leq 45^{\circ}\right)$.

The microphysics and properties of IP shocks and regions associated to them have been studied by several authors [Russell et al., 1983; Krauss-Varban et al., 2008; Wilson et al., 2009, 2012; Kajdič et al., 2012; Blanco-Cano et al., 2016; Kajdič et al., 2017] but, compared to the Earth's bow shock, we still know little about the detailed structure, ion distributions associated with these shocks, shock interaction with solar wind structures, shock reformation and rippling, etc.

In a recent study Blanco-Cano et al. [2016] showed that a variety of waves can be found upstream of IP shocks, and that extended foreshocks with suprathermal ions can be found ahead of the shocks. The characteristics and evolution of ion distributions upstream 
of an IP shock were discussed by Kajdič et al. [2017]. This study showed that different ion populations can be observed upstream of a single IP shock with $M_{A} \sim 4$. The ion distributions varied from field-aligned, gyrating to intermediate and diffuse. The diffuse ion distributions were associated with compressive ultra low frequency (ULF) waves. The field-aligned ions exhibited energies of up to $20 \mathrm{keV}$, which is much more than in the case of the Earth's bow shock, which also tends to have a higher Mach number. The authors concluded that this is due to the larger curvature radii of IP shocks which enables the particle acceleration mechanisms to act for longer time periods.

Magnetic flux ropes are commonly detected in the solar wind at $1 \mathrm{AU}$. They consist of bundles of magnetic field lines twisted around a common axis. Their durations as observed by spacecraft vary from tens of minutes to tens of hours. The most studied interplanetary flux ropes are magnetic clouds (MC) [Burlaga et al., 1981; Bothmer and Schwenn, 1998], which have large scales with diameters around 0.20-0.40 AU, and durations of $\sim 24 \mathrm{hrs}$ at the orbit of earth. MCs originate at the solar corona, being a subgroup of ICMEs. Small scale interplanetary flux ropes (known as SIFR) have diameters usually less than $0.20 \mathrm{AU}$ and durations across the spacecraft from a few minutes to a few hours [Moldwin et al., 2000; Feng et al., 2008]. In contrast to MC, SIFRs have received less attention, with only a few works focusing on them [Cartwright and Moldwin, 2010; Yu et al., 2016].

The origin of SIFR is still not totally understood, while some authors believe that they form at the sun [Feng et al., 2007; Rouillard et al., 2009], others interpret their origin in terms of magnetic reconnection at the heliospheric current sheet [Moldwin et al., 2000]. In a recent study Zheng and $H u$ [2018] explained the origin of small magnetic islands, i.e., flux ropes, in terms of intermittent solar wind turbulence.

SIFR are common in the heliosphere, as are IP shocks, so it is very probable that they can interact as they propagate through the heliosphere. As pointed out by Rouillard et al. [2009] SIFR can interact with other transient heliospheric structures, such as stream interaction regions (SIR) and shocks.

Numerous studies have shown that the interaction of solar wind discontinuities (current sheets/tangential discontinuities) with the earth's bow shock can result in the formation of foreshock transients such as hot flow anomalies [Schwartz, 1995]. There are few papers which have investigated how the earth foreshock and bow shock change when a flux rope such as a magnetic cloud (MC) crosses the shock. Turc et al. [2014, 2015] found that the Alfvén Mach number decrement due to the enhanced field inside the MC can attenuate the foreshock region and weaken the shock. They show that the foreshock can move along the bow shock surface, following the rotation of the MC's magnetic field rotation. Various studies have shown that the interaction of interplanetary shocks with magnetic clouds can compress them, and even contribute to enhancing their geomagnetic effects (e.g. Wang et al., 2003). To our knowledge no study has focused on understanding how the interaction of a SIFR or similar structure impacts IP shock structure and the surrounding upstream and downstream regions.

Shocks are well known as particle accelerators in the heliosphere [Lee et al., 2012]. IP shocks have been associated to gradual solar energetic particle (SEP) and to energetic storm particle (ESP) events. While gradual SEPs are explained in terms of acceleration of particles occurring all the way from the Sun [Reames, 2013], ESP are explained in terms of local shock acceleration [Gosling et al., 1981]. Shock ion reflection and acceleration depend on the shock geometry which, as we show below, can change when a structure with a rotating magnetic field crosses an IP shock.

In this work we present observations of the interaction of a shock with a small flux rope like structure (FRLS) at three different locations and times. This FRLS is similar to the SIFR mentioned above, although the field rotation is less than at the reported SIFR 
[see for example Moldwin et al., 2000], and its duration is smaller as we show further in the text. We investigate how this interaction can impact the shock geometry, the regions near the shock and the ion acceleration at the shock, modulating the fluxes of energetic particles. The next section describes the observations, including an overview of the data, followed by a description of ion distributions, and energetic particle associated events. The last section discusses our results and conclusions.

\section{Observations}

\subsection{Overview}

In this study we use Cluster, WIND and ACE mission observations. Cluster is a four-spacecraft mission in orbit around the earth that provides magnetic field and plasma data near our planet. We use magnetic field data from the Fluxgate Magnetometer (FGM) [Balogh et al., 2001]) and from the Cluster Ion Spectrometer (CIS) [Rème et al., 2001]. The CIS-HIA instrument provides 3-D ion distributions and moments in the energy range $5 \mathrm{eV}-32 \mathrm{keV}$ with basic time resolution at the spin period (approximately 4 second). WIND [Lepping et al., 1995] and ACE [Smith et al., 1998] are missions designed to observe the solar wind before it reaches the magnetosphere. In this work we use data from the magnetometers on board the two missions and measurements of energetic protons from the Wind 3DP (PESA) Three-Dimensional Plasma and Energetic Particle Investigation (Proton Electrostatic Analyzer) and ACE Electron, Proton, and Alpha Monitor (EPAM) instrument [Lin et al., 1995; Gold et al., 1998].

A shock was observed by Cluster 1 (C1) at 1:27:42 UT on February 18, 2011 followed by a mini FRLS shortly afterwards with a clear smooth rotation in the $B_{y}$ component. Figure 1 shows magnetic field magnitude and components, plasma temperature (parallel and perpendicular to the ambient field), HIA phase space density (PSD) omnidirectional energy spectra, and the PSD for suprathermal ions with energies $12-30 \mathrm{KeV}$. Data shown are at 22 samples per second for the magnetic field, at spin cadence for the CIS-HIA ion moments (i.e., every 4.2 seconds for $\mathrm{C} 1$ for this period), and at a cadence of every two spins ( 8.4 seconds) for the HIA energy spectra. The shock observed by $\mathrm{C} 1$ was quasi-parallel, with $\theta_{B n}=34^{\circ}$ and $B_{\mathrm{d}} / B_{\mathrm{u}}=2.75$ (subscripts u and d indicate upstream and downstream values). The magnetosonic Mach number was $M_{m s}=2.74$. HIA spectra show the presence of a $\sim 5$ minute foreshock before the shock crossing, with ions at energies above $1 \mathrm{keV}$. As we will show below, complex ion distributions permeated this region. The shock was driven by an ICME observed by C1 from 04:15 to 10:10 UT (not shown). The ICME can be classified as a MC due to the strong magnetic field inside it, the extended smooth magnetic field rotation, and the low values of plasma beta (see Figure 7 which shows the MC observed by ACE).

An interesting feature of this event is that a small flux-rope like structure was observed after the shock at 1:29:50, lasting $\sim 7 \mathrm{~min}$. The FRLS was identified by the clear smooth rotation in the $B_{y}$ component. We also considered the field rotation in azimuth and elevation angles. As the spacecraft entered the FRLS the field direction changed up to $25^{\circ}$ in the elevation angle, and $\sim 40^{\circ}$ in azimuth from the sheath average direction. We identify this structure as a flux rope-like structure and not a flux rope, because only one B component rotates smoothly and the changes in elevation and azimuth angle are smaller than in the SIFRs reported for example in Moldwin et al. [2000] with changes in elevation and azimuthal angles $>100^{\circ}$. We determined the duration of the FRLS considering the $B_{y}$ rotation and the values of azimuthal and elevation angles. There is a decrement of suprathermal ions with energies $12-30 \mathrm{KeV}$ inside the FRLS. The panels of temperature values show that $T_{\perp} / T_{\|}>1$ within the FRLS. This occurs because the structure crossed through a quasi-perpendicular shock, as we will show below when we discuss WIND and ACE observations. The bottom panel of Figure 1 shows ion phase space density for particles with energies $12-30 \mathrm{keV}$. It is clear that upstream of the shock the ener- 

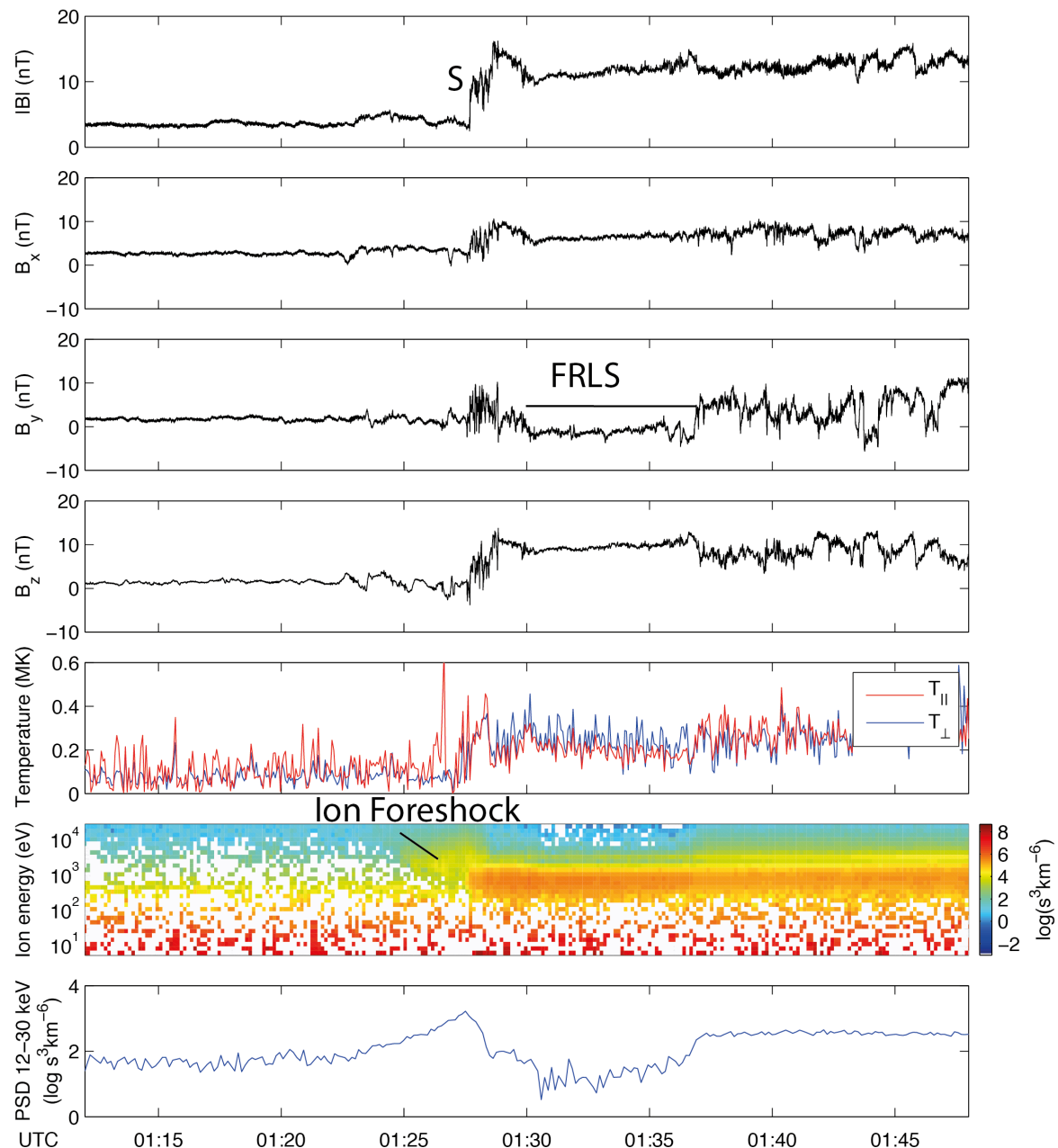

Figure 1. IP Shock and FRLS observed by Cluster 1 on February 18, 2011. The shock was observed ta 1:27:42 UT, and the FRLS was crossed from 1:29:50 to 1:36:50 UT. From top to bottom panels show magnetic field magnitude and components (GSE coordinates), parallel (red) and perpendicular (blue) plasma temperatures relative to magnetic field direction, ion phase space energy spectra, and phase space density for ions with energies in the range $12-30 \mathrm{KeV}$. The plasma data are from the CIS-HIA instrument.

getic ion population increases closer to the shock, with a peak at the shock crossing, and then drops within the FRLS. Note that because of the CIS-HIA mode for this period the solar wind beam upstream of the shock is not properly captured in the 3D omnidirectional data shown. One reason that it shows more clearly downstream is that its temperature has increased and there is a deflection of the solar wind velocity at the shock which brings a part of it into the sampling region of the instrument.

The shock and FRLS were also observed by Cluster 2 (C2), Cluster 3 (C3), and Cluster 4 (C4). The maximum spacecraft separation was $\sim 6500 \mathrm{~km}$ along $X_{G S E}, \sim 7000$ $\mathrm{km}$ along $Y_{G S E}$, and $\sim 7500 \mathrm{~km}$ along $Z_{G S E}$ (see Figure 3). Figure 2 shows magnetic field components and magnitude for all Cluster spacecraft during a $\sim 8$ min interval. It is possible to see that the region upstream of the IP shock was permeated by noncompressive irregular fluctuations. C1, C3 and C4 observed compressive whistler waves adjacent to the shock. Some trains of noncompressive whistlers can be seen further upstream, see for example $\mathrm{C} 1$ and $\mathrm{C} 2$ magnetic field components before 1:23:00 UT. 

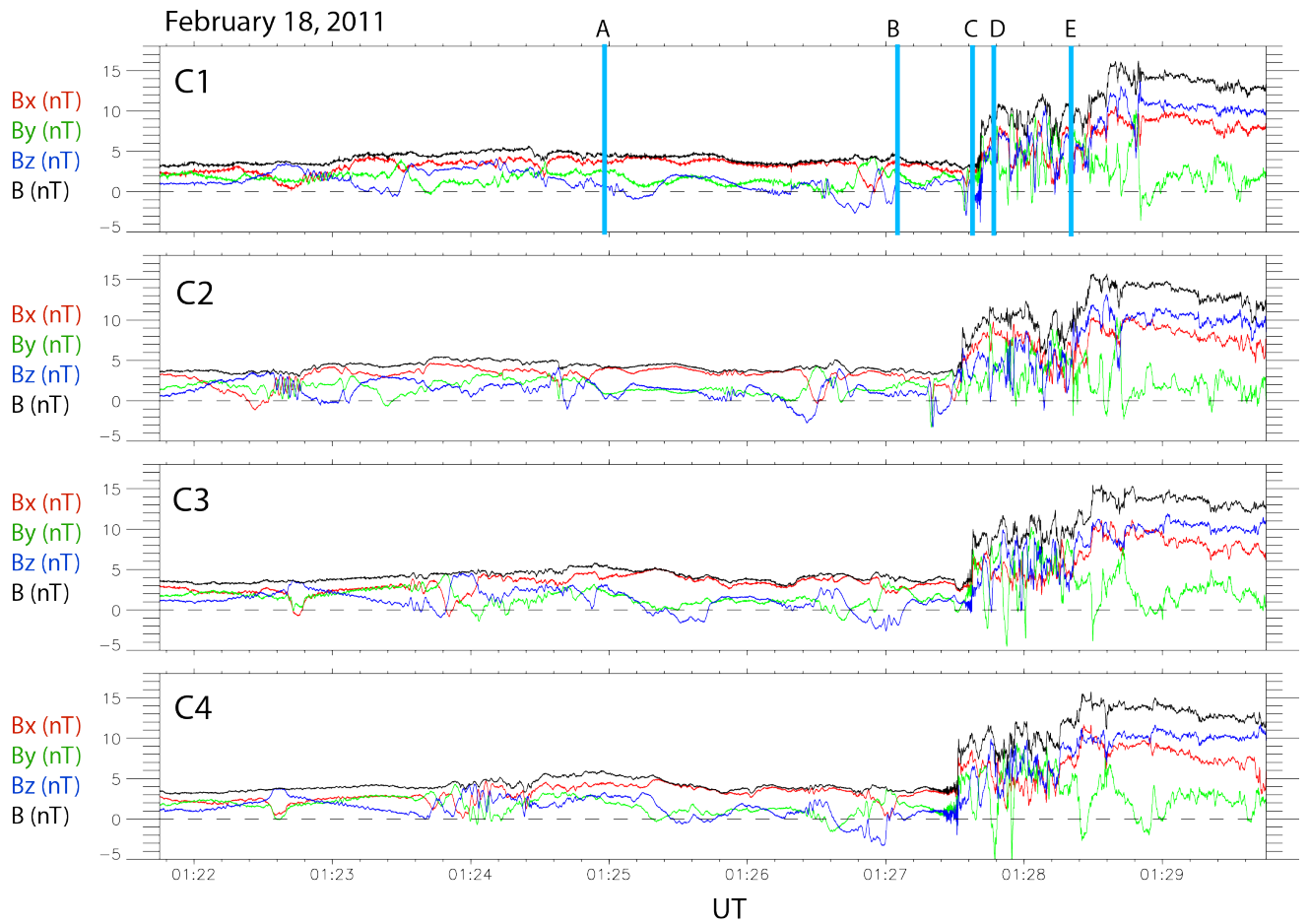

Figure 2. Magnetic field components and magnitude observed by Cluster 1 (C1), Cluster 2(C2), Cluster 3 (C3), Cluster 4 (C4) showing the upstream region, the IP shock and the downstream fluctuations observed on February 18, 2011. Lines in blue and labeled A-E indicate the times corresponding to the distributions displayed in Figure 8.

Downstream of the shock the spacecraft observed a sheath with compressive fluctuations (see Figure 2), with amplitudes reaching 5nT. After 1:29:50 when the FRLS is crossed the field is less perturbed, $B_{y}$ becomes negative, and the large amplitude magnetic field fluctuations disappear (see Figure 1).

As shown on Figure 2, the magnetic shock profile is very similar in the data of the four Cluster spacecraft. However, ACE and WIND observed the same shock at an earlier time, but with a quasi-perpendicular geometry due to the state of shock interaction with the FRLS. Figure 4 shows ACE, WIND and Cluster 2 magnetic field data at a resolution of $1 \mathrm{~s}, 93 \mathrm{~ms}$, and $45 \mathrm{~ms}$ respectively in GSE coordinates. The FRLS is shaded in yellow and a clear rotation in $B_{y}$ is observed by the three spacecraft. Figure $4 \mathrm{a}$ shows that when ACE crossed the shock, the FRLS appears as just having entered into the downstream region with a large portion still upstream. The upstream magnetic field changed $\sim 20^{\circ}$ in elevation angle and $\sim 20^{\circ}$ in azimuthal angle from the pristine solar wind into the FRLS. The shock transition is sharp as in the case of WIND (panel b), with a quasiperpendicular geometry, $\theta_{B n}=71^{\circ}$. The duration of the FRLS is around $16 \mathrm{~min}$, i.e., longer than at $\mathrm{C} 1 / \mathrm{C} 2$, where the structure is downstream from the shock. The FRLS is observed for a shorter time interval once it has been processed by the IP shock. This is due to the fact that (1) the IP shock is a fast forward shock, so the plasma and the magnetic field compress as they cross it. Both quantities thus obtain higher values but because the total FRLS mass and magnetic flux are conserved, the size of the structure must diminish. (2) In the spacecraft frame the downstream plasma (and FRLS) velocity is larger compared to the upstream value meaning that the structure will pass the spacecraft in less time. Compression of magnetic structures such as a FRLS, flux ropes, and magnetic clouds downstream of shocks has been reported in the past literature (see for example 

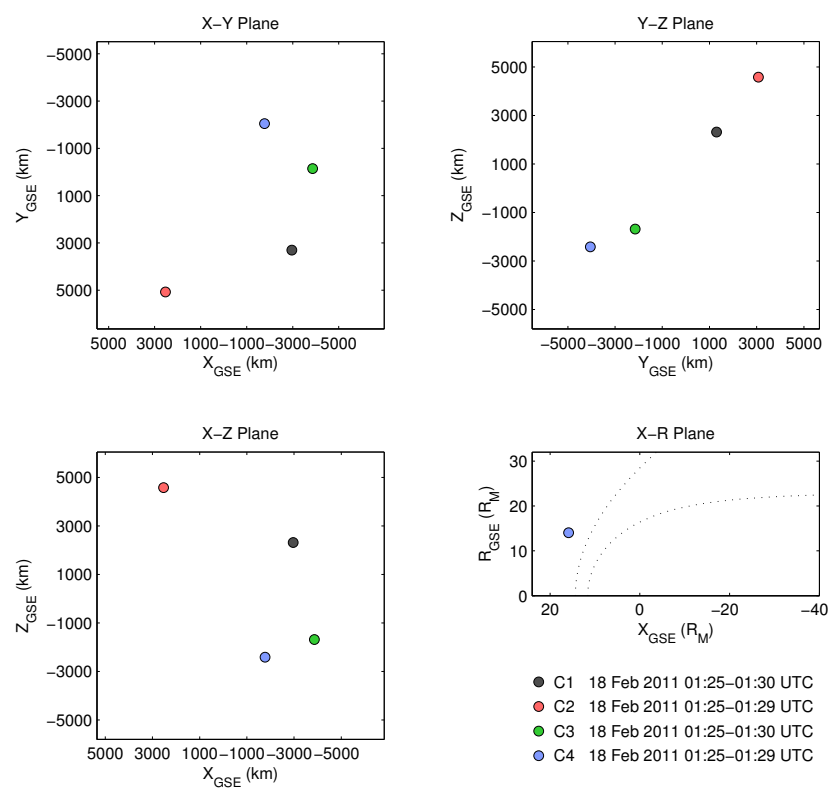

Figure 3. Average locations of C1-C4 during the interval 01:25-01:30 UT on February 18, 2011.

Wang et al. [2003]. Therefore it is very possible that the smaller size of the FRLS observed by Cluster is due to the compression that the structure suffered by crossing the shock. Compressive fluctuations are found in the rear part of the FRLS. Using the solar wind speed of $360 \mathrm{~km} / \mathrm{s}$ observed by ACE, we estimate the size of the FRLS as $51 R_{E}$ ( $0.002 \mathrm{AU})$. This is very small compared with the size of the SIFRs reported by Moldwin et al. [2000] of $191 R_{E}(0.008 \mathrm{AU})$ and is comparable to $117 R_{E}(0.005 \mathrm{AU})$, the size reported by Feng et al. [2008].

Panel $\mathrm{b}$ of Figure 4 shows that WIND observed a similar field profile to ACE data, with clear rotation only in the $B_{y}$ component and similar variations in azimuth and polar angles, around $\sim 20^{\circ}$. The FRLS is being overtaken by the shock. The shock is quasiperpendicular with $\theta_{B n}=80^{\circ}$. Similar to WIND observations, it is possible to see that the FRLS internal structure is modified as it crosses the shock, with large compressive fluctuations appearing in the rear part. The duration of the FRLS is around 13 minutes.

Panel c of Figure 4 shows the shock and FRLS as observed by Cluster 1 spacecraft. The shock transition region is more complex than for ACE and Wind, as expected for a quasi-parallel shock, with upstream fluctuations in the three field components. The magnetic field jump associated with the IP shock is $B_{d} / B_{u}=2.75$. This is smaller than at Wind $\left(B_{d} / B_{u}=3.28\right)$ and $\operatorname{ACE}\left(B_{d} / B_{u}=3.12\right)$. As mentioned earlier, the FRLS was longer in WIND and ACE data (Figure 4) and only shows fluctuations in the rear part, which is in contrast to Cluster observations. The fact that shock geometry changes due to the interaction with the FRLS is similar to the findings of Turc et al. [2015] who have shown that the values of the bow shock $\theta_{B n}$ can change when a magnetic cloud (flux rope) crosses the shock.

Figure 5 shows the location of C1, WIND, and ACE. WIND and ACE were separated around $50 R_{E}$ along $X_{G S E}$, and $\mathrm{C} 1$ was separated more than $175 R_{E}$ from them, and was closer to the earth. The largest separation of C1, WIND and ACE was around $11 R_{E}$ along $Y_{G S E}$, and $15 R_{E}$ along $Z_{G S E}$, indicating that the FRLS had at least these dimensions along these directions. 

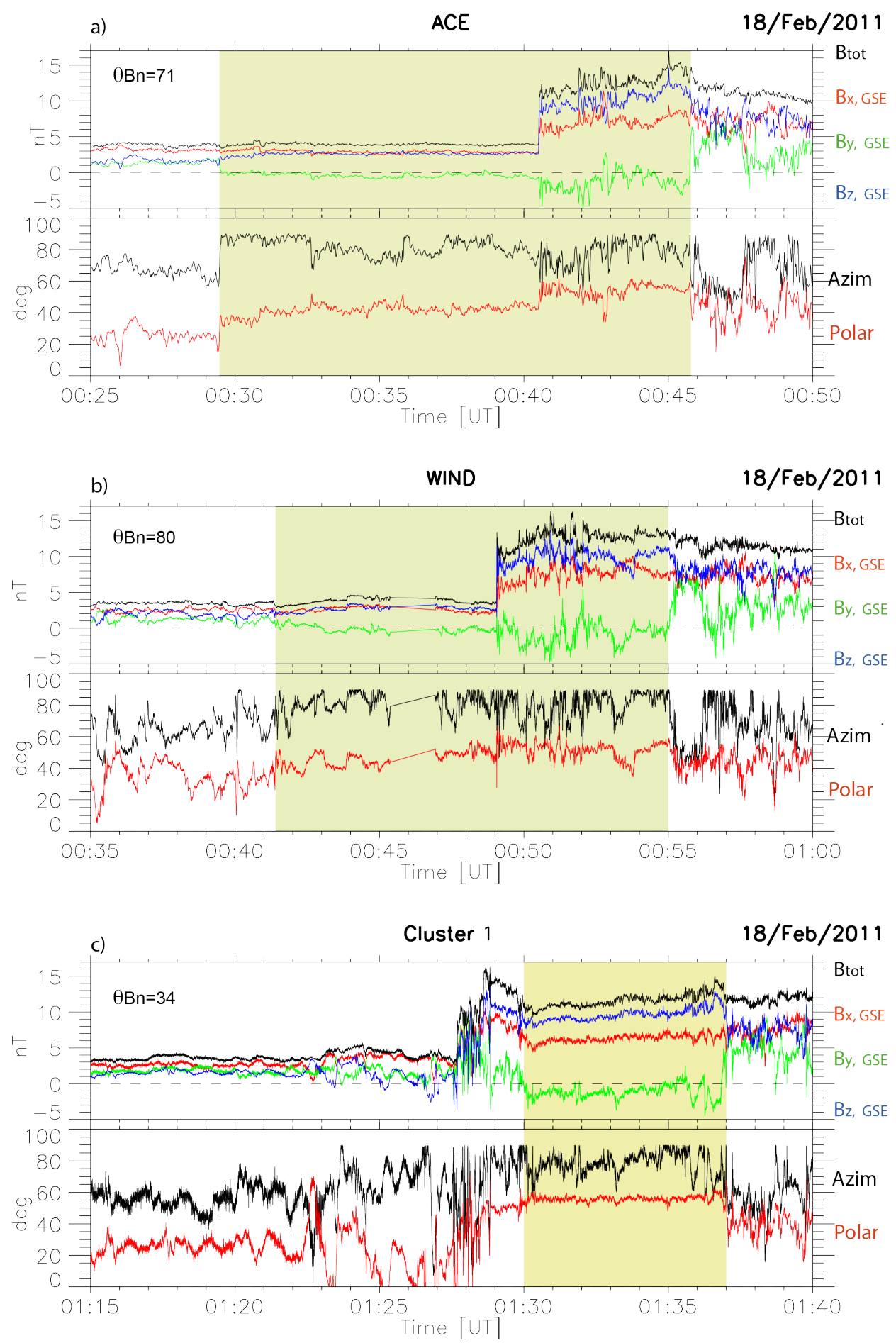

Figure 4. ACE, WIND and Cluster 1 magnetic field data showing $\mathrm{Bx}, \mathrm{By}, \mathrm{Bz}$ and $\mathrm{B}$ in GSE coordinates. Azim and Polar are the azimuthal and elevation angle of the field vector. The location of the FRLS is indicated by the yellow shade in all panels. 

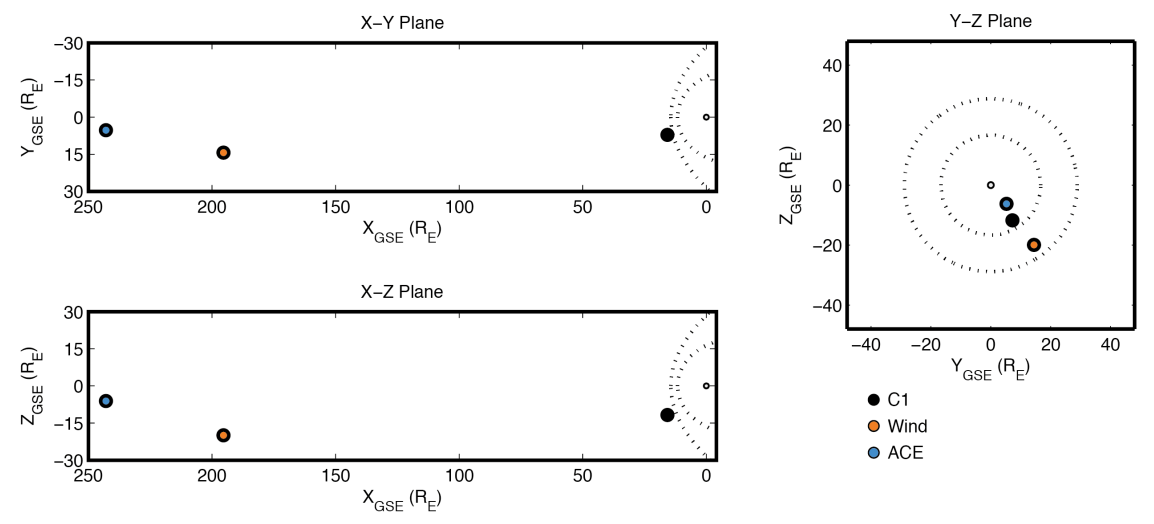

Figure 5. Average locations of $C 1$, WIND and ACE during the interval of study on February 18, 2011. The dotted lines on panels a and $\mathrm{b}$ indicate nominal locations for the magnetosphere and bow shock.

\subsection{Ion Distributions}

The fact that the shock geometry changes due to its interaction with the FRLS provides us with a good opportunity to study ion distributions recently injected into the upstream region by a newly created quasi-parallel shock using Cluster observations. Figure 2 (top panel) shows $\mathrm{C} 1$ magnetic field data upstream and through the shock, with lines in blue and lettered A-E indicating times when the ion distributions shown in Figure 6 were measured by $\mathrm{C} 1$. Note that at this time the CIS-HIA instrument was in a solar wind mode, so the 3D data shown from the energy range $5 \mathrm{eV}-32 \mathrm{keV}$ is missing the low energy part of the solar wind sector. The onboard moment data (density, velocity, etc.) are calculated with data from the solar wind sector (containing the solar wind beam) and available at spin time resolution. Velocity space cuts through the phase space distribution are shown in a frame corresponding to the ISR2 instrument frame (approximately the same as GSE) but rotated to field parallel-perpendicular coordinates. Because the instrument is in a solar wind mode the solar wind beam is mostly not sampled in these plots, but the projection of solar wind velocity from the onboard moments is plotted as a black dot.

Panels A-C show upstream distributions. Far from the shock at 1:24:59 few suprathermal ions are present, closer to the shock, at 1:27:05 wide and hot diffuse (near-isotropic) distributions are present at all times (panels B and C). Just before shock crossing, at 1:27:39 (Figure 6C) there is a significant beam superposed on the diffuse ion distribution. The beam, as seen in the $\left(V_{\perp 1}, V_{\perp 2}\right)$ plane at $V_{\|}=0$, has a significant $V_{\perp 2}=500 \mathrm{~km} \mathrm{~s}^{-1}$. It also has a considerable $V_{\|}$spread since it can be followed to increasing negative $V_{\|}$, and a part of it can be seen in the $\left(V_{\perp 1}, V_{\perp 2}\right)$ cut at $V_{\|}=-300 \mathrm{~km} \mathrm{~s}^{-1}$ (for $V_{\perp 1}>0$ ). This beam signature of gyration together with spread of parallel velocities, has been identified with bursts of ion injection from the thermal population at quasi-parallel shocks [Sundberg et al., 2016]. We should note that with incomplete 3D velocity space coverage, and relatively low time resolution it is not possible to be more definitive about the ion injection signature at this shock. But the presence of bursts of coherent beams at the same time as a diffuse population at higher energies is consistent with observations at the Earth's bow shock.

Figure 6 panels D-F show ion distributions observed by $\mathrm{C} 1$ downstream from the shock, indicated in Figure 2 (top panel). Hot ions are observed just after shock crossing 
with evidence of ion bunching (panels D-E). It is interesting to note that suprathermal ions almost disappear inside the flux rope like structure (panel F).

\subsection{Energetic particles: SEP and ESP events}

IP shocks driven by ICMEs are commonly associated with gradual SEP (solar energetic particle) [Reames, 2013] and ESP (Energetic storm particle) [Gopalswamy et al., 2003; Reames, 2013] events. While gradual SEP events are formed by particles accelerated all the way from the sun via 1st order Fermi acceleration, ESP events are related to particles accelerated locally by IP shocks, thus their peak occurs near or at shock crossing [Bryant et al., 1962].

Figure 7 shows plots of magnetic field magnitude, $B_{y}$ component and particle differential fluxes (PDF) at various energy channels for WIND (panels a and b) and ACE (panels $\mathrm{c}$ and d) during three days to show the large scale configuration of the field and energetic ions near, and at the shock under study. Panels (a) and (c) with the B-field illustrate the shock structure followed by a sheath and an ICME identified as a magnetic cloud due to the enhanced B magnitude value and smooth rotation of $B_{y}$. Proton fluxes show that the shock was associated with a gradual SEP event. The enhancements for the various energy channels are different and appear up to $\sim 42 \mathrm{hr}$ ahead of the shock. The lower energy channels 8-30 keV, and 20-58 keV for WIND, and 47-65 keV, 112-187 keV, for ACE show the development of an ESP just after shock crossing. Upstream of the shock the particle fluxes show apparently exponential decrements. This is particularly clear for the 8-30 $\mathrm{keV}$ channel observed by WIND. This shows that local shock acceleration has occurred before the FRLS interacts with the shock. The lux of energetic particles drops inside the magnetic cloud for most energy channels at WIND and ACE. The spectra corresponding to the highest energy channels, show no ions inside the MC.

Figure 8 shows zoomed-in plots of the shock region observed by Wind and ACE in the same format as Figure 7. Figure 9 shows $\mathrm{C} 1$ and $\mathrm{C} 4$ magnetic field magnitude, $\mathrm{C} 1$ CIS-HIA and C4 RAPID fluxes of suprathermal particles with energies 10-14 keV, 25-34 $\mathrm{keV}$, and of particles observed at energy channels 42,92 and $160 \mathrm{keV}$. It is clear that the interaction of the FRLS with the IP shock reported here has an impact on the spectra of energetic ions observed near and at shock crossing. While Wind and ACE observed just a narrow peak associated with an ESP behind the shock, Cluster spectra showed evidence of an extended foreshock region filled with locally accelerated particles with energies up to $160 \mathrm{KeV}$.

The PDFs observed by WIND and ACE show a drop at the lower energy channels just before the shock crossing. Enhancements in the energetic ions flux are observed behind the shock at three WIND energy channels $8-30 \mathrm{keV}, 20-58 \mathrm{keV}$ and $58-125 \mathrm{keV}$. There is indication of few ions at $115-400 \mathrm{keV}$ behind he shock. It is interesting to note that the passage of the FRLS through the shock results in a decrement in the amount of energetic ions. This indicates that the interaction of FRLS with the IP shock locally inhibits the ion acceleration, and can have an effect in the energetic particle fluxes or PDFs causing a depletion of energetic ions.

In the case of ACE (Figures 7 and 8), energetic ions with energies $47-65 \mathrm{keV}$ are present upstream of the shock ahead of the FRLS but the flux of these ions decreases at the FRLS. The flux reaches a peak $\sim 5.5 \times 10^{5} \mathrm{~cm}^{2} \mathrm{~s}$ ster Mev a few minutes after shock passage. The peak at channel $112-187 \mathrm{keV}$ reaches a smaller value $\sim 1.0 \times 10^{5} \mathrm{~s}$ ster Mev after the shock and FRLS passage, with no enhancement upstream. This shows that local shock acceleration is occurring and limited to lower energies.

Some inferences can be made from the void of energetic particles in the FRLS. When the shock is locally quasi-perpendicular the acceleration is not efficient, certainly less efficient than for the quasi-parallel configuration, despite any possible pre-existing en- 

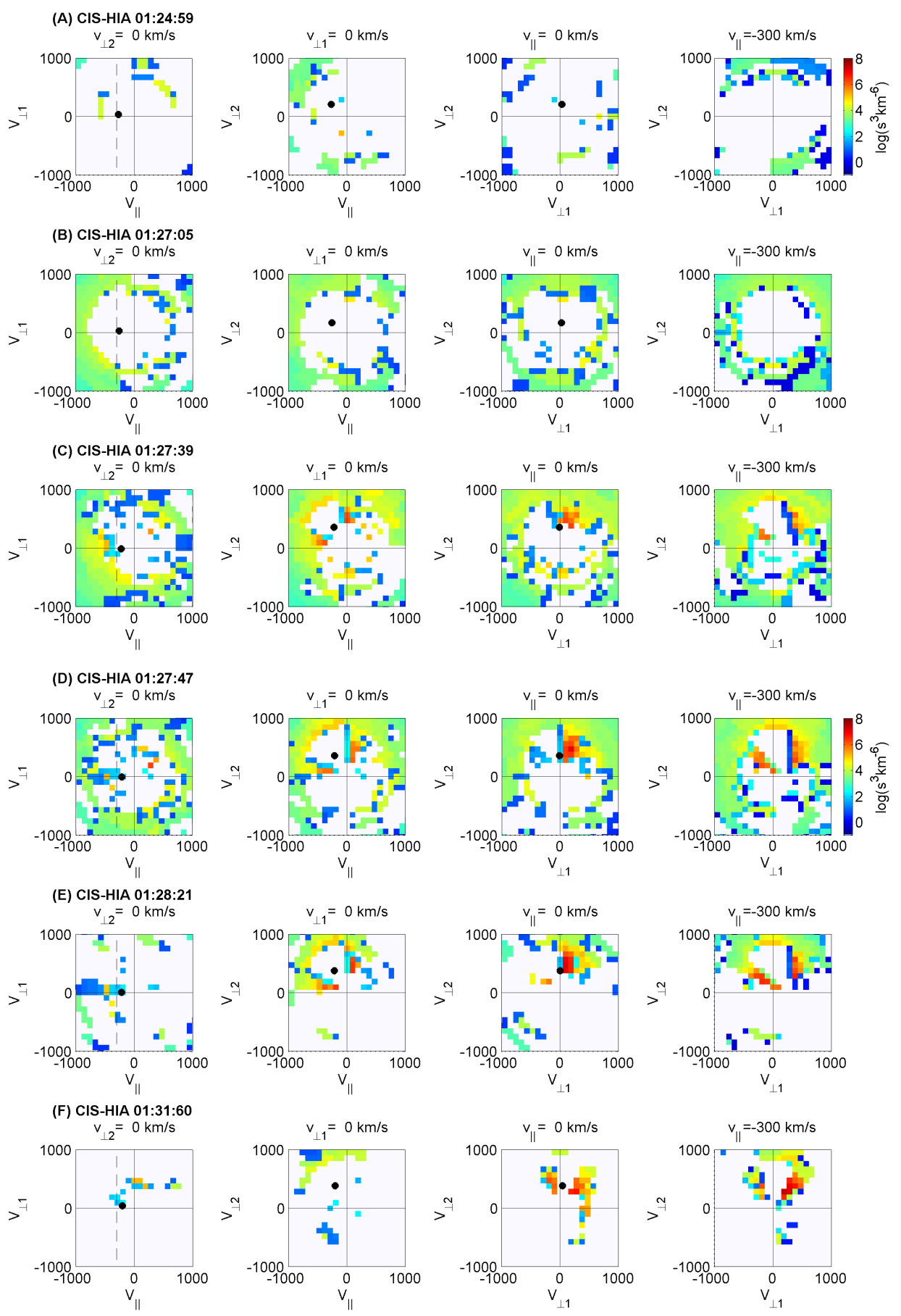

Figure 6. CIS-HIA ion velocity space distributions for times indicated in Figure 2 and corresponding to the upstream region (A-B), just before shock crossing (C), downstream (D-E), and inside the FRLS (F). Each panel shows cuts on the planes $\left(V_{\|}, V_{\perp 1}\right),\left(V_{\|}, V_{\perp 2}\right)$ and $\left(V_{\perp 1}, V_{\perp 2}\right)$ at $V_{\|}=0$, and the $\left(V_{\perp 1}, V_{\perp 2}\right)$ plane at $V_{\|}=-300 \mathrm{~km} / \mathrm{s}$, i.e., the solar wind parallel velocity. Due to instrument mode the solar wind is not sampled, but the solar wind velocity from the onboard moments is plotted as a black dot. Distributions are shown in the ISR2 instrument frame (close to GSE) rotated to field parallel-perpendicular coordinates. 

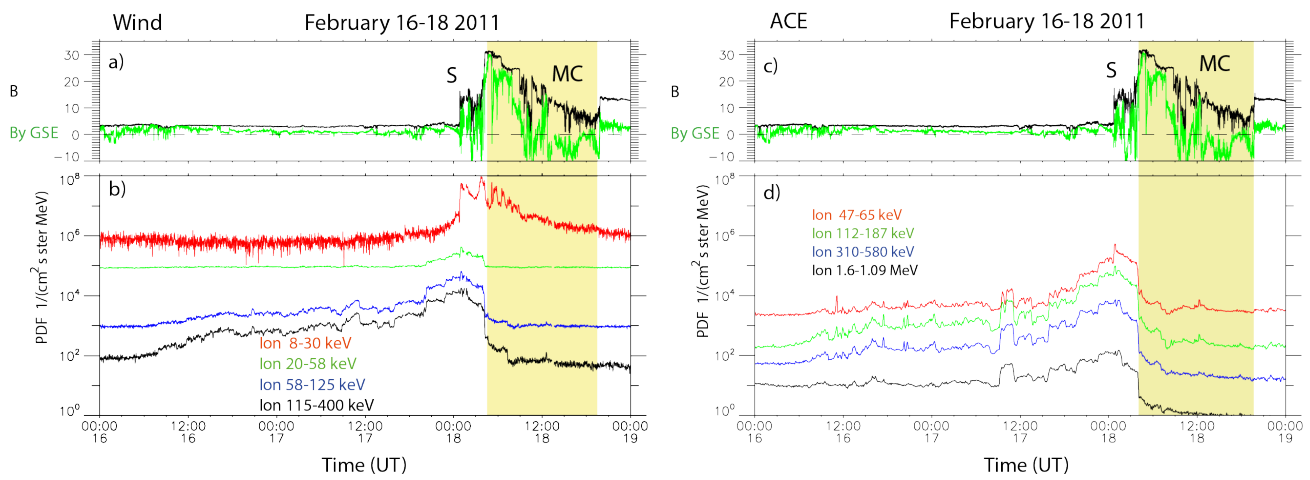

Figure 7. Magnetic field magnitude, By component and energetic particles PDF observed by Wind and ACE on days February 16-18, 2011. The various energy channels are indicated in the figure.
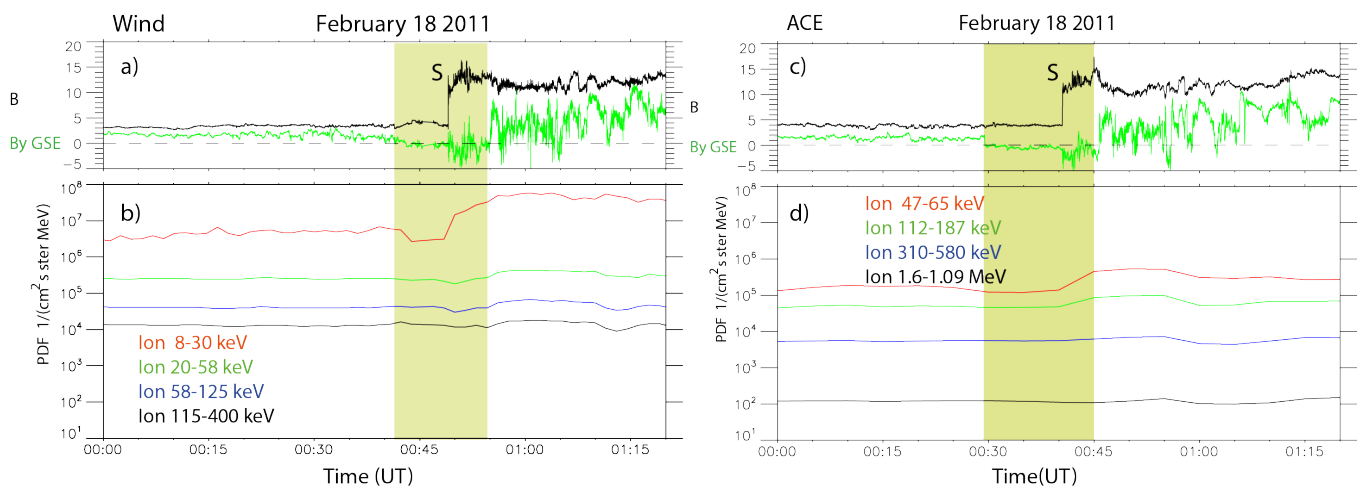

Figure 8. Magnetic field magnitude, $B_{y}$ component and energetic particles PDF observed by WIND and ACE during 80 minutes on February 18, 2011. The various energy channels are indicated in the figure.

ergetic component ahead of the shock. Further, the gradient of energetic particles across the FRLS boundaries shows that they are effective barriers for the energetic particles. This indicates that cross-field diffusion is not effective, but also, if parallel propagation is dominant, then it indicates that the field lines within the FRLS do not connect to any region with high fluxes of energetic particles. Given the scale of IP shocks this might be evidence of the interesting idea that the observed FRLS, although of small transverse scale, might have a much larger scale along its axis.

In contrast, the energetic particles observed by Cluster have a very different behaviour. Due to the Cluster orbit, no SEP event is observed. Figure 9 shows $\mathrm{C} 1$ and C4 magnetic field magnitude, C1 CIS-HIA and C4 RAPID flux densities of suprathermal particles with energies $10-14 \mathrm{keV}, 25-34 \mathrm{keV}$, and of particles observed at energy channels 42, 92 and $160 \mathrm{keV}$. Panels b and c show a ESP event with a peak in the energetic particle flux at the time of the shock. The peak intensity is largest for particles with lower energies $10-14 \mathrm{keV}$ measured by $\mathrm{C} 1$, reaching almost $2.65 \times 10^{5} \mathrm{keV} / \mathrm{cm}^{2} \mathrm{~s}$ ster $\mathrm{keV}$. Flux enhancements appear around fifty minutes ahead of the shock indicating an ion foreshock and that particles can diffuse in the region. The extension of the foreshock appears larger as observed by $\mathrm{C} 1$ than by $\mathrm{C} 4$. For particles with energies $25-34 \mathrm{keV}$ the peak reaches $1.5 \times 10^{5} \mathrm{keV} / \mathrm{cm}^{2} \mathrm{~s}$ ster $\mathrm{keV}$. The enhancement in density for these two ranges of energies extends $\sim 10$ and $\sim 23$ min ahead of the shock. In contrast, the peaks in the density observed by $\mathrm{C} 4$ at energies 42,92 and $160 \mathrm{keV}$ reach $2.15 \times 10^{4}, 5 \times 10^{3}$ and $1 \times 10^{3}$ respectively. Accelerated particles at 42 and $92 \mathrm{keV}$ are observed up to $50 \mathrm{~min}$ ahead of 


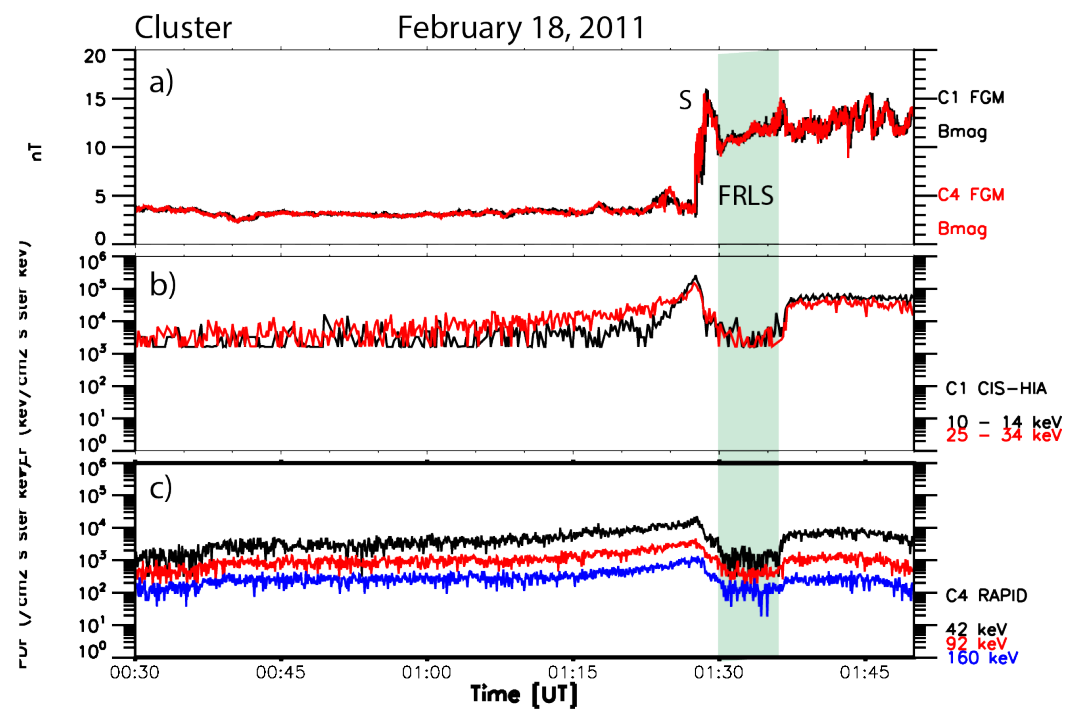

Figure 9. Magnetic field magnitude, and energetic ions (particle energy flux) observed by C1 (panel b) and C4 particle differential flux (panel c) spacecraft.

the shock. The upstream fluxes at all energies decrease exponentially in agreement with shock acceleration from the thermal solar wind population as predicted by Lee [1983]. It is interesting to see that no energetic ions are observed inside the FRLS in any of the channels.

\section{Discussion and Conclusions}

Using data from Cluster, Wind and ACE missions we have shown that the interaction of a relatively small scale flux rope-like structure (FRLS) with an IP shock can locally change the shock geometry and influence the spectra of energetic particles. Wind and ACE observed a quasi-perpendicular shock at the time when the FRLS was crossing it, and Cluster observed a quasi-parallel shock with the FRLS on the downstream side. The change in shock geometry affects ion injection processes, particle acceleration, and the upstream and downstream regions. Shock geometry affects the motion of reflected ions, and this in turn affects particle injection, acceleration and wave generation upstream. When the shock is quasi-parallel $\left(\theta_{B n}<45^{\circ}\right)$ the reflected particles can escape upstream producing a complex and extended shock structure, and a foreshock region ahead of the shock where various suprathermal ion distributions and waves can exist. When the shock geometry is quasi-perpendicular $\left(\theta_{B n}>45^{\circ}\right)$, some ions can escape upstream, but others are turned around by the magnetic field and sent back to the shock; in this case the shock transition is less extended and no wave foreshock is produced. Shock geometry can also affect shock heating. Quasi-perpendicular shocks can heat the plasma more efficiently in the direction perpendicular to the magnetic field, leading to temperature anisotropy $\left(T_{\perp} / T_{\|}>1\right)$ downstream of them, as can be observed inside the FRLS observed by Cluster.

Ion injection, wave generation and acceleration processes are also affected by the fact that IP shocks are not planar and that their structure is not smooth. Using data from three spacecraft, Szabo et al. [2001] and Szabo [2005] showed evidence of significant shock surface irregularities on spatial scales between $\sim 10-80 R_{E}$. They found that smaller and slower magnetic clouds can drive more corrugated shocks. In addition, several 
works using hybrid simulations [Winske and Quest, 1988; Lowe and Burgess, 2003; Ofman and Gedalin, 2013] have shown that shock rippling occurs due to instability and/or surface waves inherent to the shock when the Alfvén Mach number $M_{A}$ is $>4.7$. The wavelength of this rippling is of the order of the ion inertial length. Ripples in quasi-perpendicular shocks can also be produced by the interaction of upstream Alfvén waves with the shock [Lu et al., 2009]. These authors performed 2D hybrid simulations of the interaction of a perpendicular shock with upstream Alfvén waves as a proxy for magnetic turbulence. The resultant shock has an irregular shape, and is a mixture of planar shocks with different $\theta_{B n}$. Quasi-parallel or marginally quasi-perpendicular shock surfaces can also experience irregularities with much larger wavelengths ( $\sim 100$ ion inertial lengths) due to upstream wave impact on the shock [Krauss-Varban et al., 2008], that can change the local $\theta_{B N}$. This was shown observationally by Kajdič et al. [2019] who observed that even moderate $\mathrm{M}_{A}$ (3.5-4.4) and relatively high $\beta$ (1.8-3.6) IP shocks may have irregular surfaces and that these irregularities cause shock profiles to vary even at small spatial scales ( $\geq 5$ ion inertial lengths). The consequences of shock rippling on upstream ions has also been recently studied by Hao et al. [2016]. These authors show that the reflection or downstream transmission of upstream ions depends on their interaction with different parts of the ripples.

In this work we have shown that shock fronts can also change locally due to the interaction with small scale solar wind structures such as a FRLS. Considering that in the case of our event the largest separation distance between C1, WIND and ACE was around $12 R_{E}$ along Y, and 20 along Z, we conclude that the FRLS had at least these dimensions and the related changes in shock structure must have similar scales, i.e., the FRLS changes the shock on scales much larger than an ion gyroradius of the thermal protons $(\sim 100$ $\mathrm{km}$ ). Most models of shock acceleration consider uniform shock conditions, however, as we have shown, IP shock structure can be modified by small transients. Interactions such as the one we describe may occur at various parts of the shock front and at different radial distances resulting in anisotropic suprathermal ion foreshocks, and in modulation of the energetic particles produced locally at the shock, associated with ESP events.

The ion foreshock observed by Cluster has complex ion distributions including beam and diffuse ions. This is in agreement with previous observations by Kajdič et al. [2017] who described a variety of ion distributions upstream of a single IP shock in the data of the ARTEMIS [Angelopoulos, 2011] spacecraft. These authors observed upstream ion distributions that changed from initial field-aligned (ARTEMIS-1) or gyrating (ARTEMIS2) to intermediate and diffuse distributions. The latter were observed together with compressive B-field fluctuations in the ultra-low frequency range. It was also found that fieldaligned beams exhibited much higher energies than in the case of the Earth's bow-shock.

Different ion populations are also consistent with observations of the ion injection signatures for the terrestrial quasi-parallel bow shock [Sundberg et al., 2016]. ULF waves are commonly observed upstream of quasi-parallel IP shocks [Blanco-Cano et al., 2016]. However, the upstream region observed by Cluster did not show well defined waves. This might be related to the fact that Cluster observes a newly formed quasi-parallel shock, and wave generation and growth has not had enough time to develop. Quasi-parallel shocks are complex structures whose dynamics depends mainly on ion time scales, after the quasiparallel shock is formed ion reflection starts to occur, however, waves need some time to grow and be observable.

Wind and ACE data show that the IP shock was related to a SEP event with evidence of an ESP event also. A drop out in the energetic particle fluxes is observed during a few minutes coinciding with the FRLS crossing the shock. This gives evidence of how a small magnetic structure can modulate the spectra of energetic particles observed at 1 AU. The occurrence of drop outs in the impulsive SEP fluxes has been reported in the past [Mazur et al., 2000]. However such drop outs can last several hours, with a mean duration of $3 \mathrm{hrs}$, and are mostly associated with impulsive SEPs, i.e., non gradual events. 
The origin of these drop outs has been interpreted in terms of a filamentary distribution of magnetic connection to the particle source [Giacalone et al., 2000]

It is interesting to note that energetic ions were not present (or were at least strongly suppressed) inside the FRLS downstream from the shock observed by CLUSTER and suppressed relative to the surrounding regions when seen upstream. This suggests that the magnetic field lines inside the FRLS are totally disconnected from the shock. In contrast to our results Zhao et al. [2018] have related small flux ropes to particle acceleration downstream from IP shocks. However, the physical situation they describe is very different to the observations we have discussed, with the presence of numerous flux ropes or magnetic islands in a region where enhancements in energetic particles are explained in terms of stochastic acceleration due to the interacting islands. Energetic particles, namely electrons have also been found inside contracting magnetic islands formed by reconnection in Earth's magnetosphere, see for example the observational study of Chen et al. [2008] and the 2D simulation results of Fu and Lu [2006] and Drake et al. [2006], among others. Energization within these closed contracting islands is explained in terms of Fermi acceleration of trapped particles. A possible explanation for the discrepancy between our results and the energetic particles found inside closed magnetic islands, is the fact that due to the 3D nature of the FRLS, there is an axial component of the field, i.e., the flux rope is not a closed structure and particles can escape from it. Additionally, the plasma inside the FRLS passes through the shock so it has to be heated. Thus, conditions differ from the closed magnetic island within reconnection regions.

$\mathrm{MC}$ are large scale flux ropes with clear magnetic field rotations. It is expected that similar effects as the ones described here take place when a MC interacts with an IP shock or a planetary bow shock. It will be part of future studies to understand in detail how MC-shock interaction modifies shock structure and particle acceleration in the case of IP shocks and the Earth's bow shock.

The Parker Solar Probe and Solar Orbiter missions will be helpful to study IP shocks closer to the Sun and interactions with heliospheric magnetic structures such as the one discussed in this manuscript. Cartwright and Moldwin [2010] found that the occurrence rate of small-scale flux ropes is slightly higher in the inner heliosphere than in the outer heliosphere. It is probable that more small scale transients which have been associated to flux ropes, such as blobs [Sheeley et al., 1997; Rouillard et al., 2011] are observed at small heliospheric distances modifying shock structure, particle injection and acceleration processes.

\section{Acknowledgments}

We acknowledge support from the Royal Society Newton International Exchange Scheme (Mexico) grant NI150051. DB acknowledges partial support from STFC (UK) grant ST/P000622/1. XBC thanks UNAM PAPIIT-DGAPA (IN105218-3) and CONACyT (255203) grants. PK's work was supported by PAPIIT grant IA101118.

We thank the Cluster instrument teams and the Cluster Science Archive (https://www.cosmos.esa.int/web/csa) for providing publically available Cluster data. We acknowledge use of clweb (http://clweb.irap.omp.eu/) written and made available by Emmanuel Penou for access to and visualization of publically available Cluster data. We thank the ACE instrument teams and the ACE Science Center for providing ACE data (http://www.srl.caltech.edu/ACE/ASC/). We thank the WIND instrument teams and NASA's Space Physics Data Facility (SPDF) for providing WIND data (https://spdf.gsfc.nasa.gov/).

\section{References}

Angelopoulos, V. (2011), The artemis mission, Space Science Reviews, 165(1), 3-25, doi: 10.1007/s11214-010-9687-2. 
Balogh, A., C. M. Carr, M. H. Acuña, M. W. Dunlop, T. J. Beek, P. Brown, K.-H. Fornaçon, E. Georgescu, K.-H. Glassmeier, J. Harris, G. Musmann, T. Oddy, and K. Schwingenschuh (2001), The Cluster Magnetic Field Investigation: overview of in-flight performance and initial results, Annales Geophysicae, 19, 1207-1217, doi: 10.5194/angeo-19-1207-2001.

Blanco-Cano, X., P. Kajdič, E. Aguilar-Rodríguez, C. T. Russell, L. K. Jian, and J. G. Luhmann (2016), Interplanetary shocks and foreshocks observed by stereo during 2007âĂŞ2010, Journal of Geophysical Research: Space Physics, 121(2), 992-1008, doi: 10.1002/2015JA021645, 2015JA021645.

Bothmer, V., and R. Schwenn (1998), The structure and origin of magnetic clouds in the solar wind, Annales Geophysicae, 16(1), 1-24, doi:10.1007/s00585-997-0001-x.

Bryant, D. A., T. L. Cline, U. D. Desai, and F. B. McDonald (1962), Explorer 12 Observations of Solar Cosmic Rays and Energetic Storm Particles after the Solar Flare of September 28, 1961, Journal of Geophysical Research, 67, 4983-5000, doi: 10.1029/JZ067i013p04983.

Burlaga, L., E. Sittler, F. Mariani, and R. Schwenn (1981), Magnetic loop behind an interplanetary shock: Voyager, helios, and imp 8 observations, Journal of Geophysical Research: Space Physics, 86(A8), 6673-6684, doi:10.1029/JA086iA08p06673.

Cartwright, M. L., and M. B. Moldwin (2010), Heliospheric evolution of solar wind small-scale magnetic flux ropes, Journal of Geophysical Research: Space Physics, 115(A8), n/a-n/a, doi:10.1029/2009JA014271, a08102.

Chen, L. J., A. Bhattacharjee, P. A. Puhl-Quinn, H. Yang, N. Bessho, S. Imada, S. MÃijhlbachler, P. W. Daly, B. Lefebvre, Y. Khotyaintsev, A. Vaivads, A. Fazakerley, and E. Georgescu (2008), Observation of energetic electrons within magnetic islands, Nature Physics, 4, 19 EP, doi:10.1088/0004-637x/706/1/687.

Drake, J. F., M. Swisdak, H. Che, and M. A. Shay (2006), Electron acceleration from contracting magnetic islands during reconnection, Nature, 443(7111), 553-556.

Feng, H. Q., D. J. Wu, and J. K. Chao (2007), Size and energy distributions of interplanetary magnetic flux ropes, Journal of Geophysical Research: Space Physics, 112(A2), n/a-n/a, doi:10.1029/2006JA011962, a02102.

Feng, H. Q., D. J. Wu, C. C. Lin, J. K. Chao, L. C. Lee, and L. H. Lyu (2008), Interplanetary small- and intermediate-sized magnetic flux ropes during 1995âĂŞ2005, Journal of Geophysical Research: Space Physics, 113(A12), n/a-n/a, doi:10.1029/2008JA013103, a12105.

$\mathrm{Fu}, \mathrm{X}$., and Q. Lu (2006), The process of electron acceleration during magnetic reconnection, Physics of Plasmas, 13, doi:10.1063/1.2164808.

Giacalone, J., J. R. Jokipii, and J. E. Mazur (2000), Small-scale gradients and large-scale diffusion of charged particles in the heliospheric magnetic field, The Astrophysical Journal Letters, 532(1), L75.

Gold, R., S. Krimigis, and S. Hawkins (1998), Electron, proton and alpha monitor on the advanced composition explorer spacecraft, Space Sci. Rev., 86(541).

Gonzalez, W., B. Tsurutani, and A. ClÃža de Gonzalez (1999), Interplanetary origin of geomagnetic storms, Space Science Reviews, 88(529).

Gopalswamy, N., S. Yashiro, A. Lara, M. L. Kaiser, B. J. Thompson, P. T. Gallagher, and R. A. Howard (2003), Large solar energetic particle events of cycle 23: A global view, Geophysical Research Letters, 30(12), n/a-n/a, doi:10.1029/2002GL016435, 8015.

Gosling, J. T., J. R. Asbridge, S. J. Bame, W. C. Feldman, R. D. Zwickl, G. Paschmann, N. Sckopke, and R. J. Hynds (1981), Interplanetary ions during an energetic storm particle event: The distribution function from solar wind thermal energies to 1.6 mev, Journal of Geophysical Research: Space Physics, 86(A2), 547-554, doi: 10.1029/JA086iA02p00547.

Hao, Y., B. Lembege, Q. Lu, and F. Guo (2016), Formation of downstream high-speed jets by a rippled nonstationary quasi-parallel shock: 2-D hybrid simulations, J. Geophys. Res., 121, 2080-2094, doi:10.1002/2015JA021419. 
Kajdič, P., L. Preisser, X. Blanco-Cano, D. Burgess, and D. Trotta (2019), First observations of irregular surface of interplanetary shocks at ion scales by cluster, The Astrophysical Journal, 874(2), L13, doi:10.3847/2041-8213/ab0e84.

Kajdič, P., X. Blanco-Cano, E. Aguilar-Rodriguez, C. T. Russell, L. K. Jian, and J. G. Luhmann (2012), Waves upstream and downstream of interplanetary shocks driven by coronal mass ejections, Journal of Geophysical Research: Space Physics, 117(A6), n/an/a, doi:10.1029/2011JA017381, a06103.

Kajdič, P., H. Hietala, and X. Blanco-Cano (2017), Different types of ion populations upstream of the 2013 october 8 interplanetary shock, The Astrophysical Journal Letters, 849(2), L27.

Krauss-Varban, D., Y. Li, and J. G. Luhmann (2008), Ion acceleration at the earth-

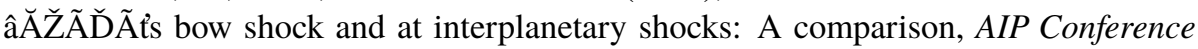
Proceedings, 1039(1), 307-313, doi:10.1063/1.2982463.

Lee, M. A. (1983), Coupled hydromagnetic wave excitation and ion acceleration at interplanetary traveling shocks, Journal of Geophysical Research: Space Physics, 88(A8), 6109-6119, doi:10.1029/JA088iA08p06109.

Lee, M. A., R. A. Mewaldt, and J. Giacalone (2012), Shock acceleration of ions in the heliosphere, Space Science Reviews, 173(247).

Lepping, R., M. Acuna, and L. e. a. Burlaga (1995), The wind magnetic field investigation, Space Science Reviews, 71(207), doi:https://doi.org/10.1007/BF00751330.

Lin, R., Anderson, and S. K.A., Ashford (1995), c, Space Science Reviews, 71(125).

Lowe, R. E., and D. Burgess (2003), The properties and causes of rippling in quasiperpendicular collisionless shock fronts, Annales Geophysicae, 21, 671-679, doi: 10.5194/angeo-21-671-2003.

Lu, Q., Q. Hu, and G. P. Zank (2009), The interaction of alfvén waves with perpendicular shocks, 706(1), 687-692, doi:10.1088/0004-637x/706/1/687.

Mazur, J. E., G. M. Mason, J. R. Dwyer, J. Giacalone, J. R. Jokipii, and E. C. Stone (2000), Interplanetary magnetic field line mixing deduced from impulsive solar flare particles, The Astrophysical Journal Letters, 532(1), L79.

Moldwin, M. B., S. Ford, R. Lepping, J. Slavin, and A. Szabo (2000), Small-scale magnetic flux ropes in the solar wind, Geophysical Research Letters, 27(1), 57-60, doi: 10.1029/1999GL010724.

Ofman, L., and M. Gedalin (2013), Two-dimensional hybrid simulations of quasiperpendicular collisionless shock dynamics: Gyrating downstream ion distributions, Journal of Geophysical Research (Space Physics), 118, 1828-1836, doi: 10.1029/2012JA018188.

Reames, D. V. (2013), The two sources of solar energetic particles, Space Science Reviews, 175(53).

Rème, H., C. Aoustin, J. M. Bosqued, I. Dandouras, B. Lavraud, J. A. Sauvaud, A. Barthe, J. Bouyssou, T. Camus, O. Coeur-Joly, A. Cros, J. Cuvilo, F. Ducay, Y. Garbarowitz, J. L. Medale, E. Penou, H. Perrier, D. Romefort, J. Rouzaud, C. Vallat, D. Alcaydé, C. Jacquey, C. Mazelle, C. d’Uston, E. Möbius, L. M. Kistler, K. Crocker, M. Granoff, C. Mouikis, M. Popecki, M. Vosbury, B. Klecker, D. Hovestadt, H. Kucharek, E. Kuenneth, G. Paschmann, M. Scholer, N. Sckopke, E. Seidenschwang, C. W. Carlson, D. W. Curtis, C. Ingraham, R. P. Lin, J. P. McFadden, G. K. Parks, T. Phan, V. Formisano, E. Amata, M. B. Bavassano-Cattaneo, P. Baldetti, R. Bruno, G. Chionchio, A. Di Lellis, M. F. Marcucci, G. Pallocchia, A. Korth, P. W. Daly, B. Graeve, H. Rosenbauer, V. Vasyliunas, M. McCarthy, M. Wilber, L. Eliasson, R. Lundin, S. Olsen, E. G. Shelley, S. Fuselier, A. G. Ghielmetti, W. Lennartsson, C. P. Escoubet, H. Balsiger, R. Friedel, J.-B. Cao, R. A. Kovrazhkin, I. Papamastorakis, R. Pellat, J. Scudder, and B. Sonnerup (2001), First multispacecraft ion measurements in and near the earth's magnetosphere with the identical cluster ion spectrometry (cis) experiment, Annales Geophysicae, 19(10/12), 1303-1354, doi:10.5194/angeo-19-13032001. 
Rouillard, A. P., N. P. Savani, J. A. Davies, B. Lavraud, R. J. Forsyth, S. K. Morley, A. Opitz, N. R. Sheeley, L. F. Burlaga, J.-A. Sauvaud, K. D. C. Simunac, J. G. Luhmann, A. B. Galvin, S. R. Crothers, C. J. Davis, R. A. Harrison, M. Lockwood, C. J. Eyles, D. Bewsher, and D. S. Brown (2009), A multispacecraft analysis of a smallscale transient entrained by solar wind streams, Solar Physics, 256(1), 307-326, doi: 10.1007/s11207-009-9329-6.

Rouillard, A. P., N. R. Sheeley, Jr., T. J. Cooper, J. A. Davies, B. Lavraud, E. K. J. Kilpua, R. M. Skoug, J. T. Steinberg, A. Szabo, A. Opitz, and J.-A. Sauvaud (2011), The Solar Origin of Small Interplanetary Transients, The Astrophysical Journal, 734, 7, doi:10.1088/0004-637X/734/1/7.

Russell, C. T., E. J. Smith, B. J. Tsurutani, J. G. Gosling, and S. J. Bame (1983), Multiple spacecraft observations of interplanetary shocks four spacecraft determination of shock normals, Solar Wind Five, NASA Conf. Publ., 2280, 385-400.

Schwartz, S. J. (1995), Hot flow anomalies near the Earth's bow shock, Adv. Space Res., 15, 107-116, doi:10.1016/0273-1177(95)00025-A.

Sheeley, N. R., Jr., Y.-M. Wang, S. H. Hawley, G. E. Brueckner, K. P. Dere, R. A. Howard, M. J. Koomen, C. M. Korendyke, D. J. Michels, S. E. Paswaters, D. G. Socker, O. C. S. Cyr, D. Wang, P. L. Lamy, A. Llebaria, R. Schwenn, G. M. Simnett, S. Plunkett, and D. A. Biesecker (1997), Measurements of flow speeds in the corona between 2 and 30râŸL, The Astrophysical Journal, 484(1), 472-478, doi:10.1086/304338.

Smith, C., J. L'Heureux, N. Ness, M. Acuña, L. Burlaga, and J. Scheifele (1998), The ace magnetic fields experiment, Space Science Reviews, 86(1), 613-632, doi: 10.1023/A:1005092216668.

Sundberg, T., C. T. Haynes, D. Burgess, and C. X. Mazelle (2016), Ion Acceleration at the Quasi-parallel Bow Shock: Decoding the Signature of Injection, The Astrophysical Journal, 820, 21, doi:10.3847/0004-637X/820/1/21.

Szabo, A. (2005), MultiâĂ ̌̌spacecraft observations of interplanetary shocks, AIP Conference Proceedings, 781(1), 37-41, doi:10.1063/1.2032672.

Szabo, A., R. P. Lepping, J. Merka, C. W. Smith, and R. M. Skoug (2001), The evolution of interplanetary shocks driven by magnetic cloud, in Solar Encounter, Proceedings of the First Solar Orbiter Workshop, vol. ESA SP-493, edited by B. Battrick, pp. 385-400.

Turc, L., D. Fontaine, P. Savoini, and E. K. J. Kilpua (2014), A model of the magnetosheath magnetic field during magnetic clouds, Annales Geophysicae, 32(2), 157-173, doi:10.5194/angeo-32-157-2014.

Turc, L., D. Fontaine, P. Savoini, and R. Modolo (2015), 3D hybrid simulations of the interaction of a magnetic cloud with a bow shock, Journal of Geophysical Research (Space Physics), 120(8), 6133-6151, doi:10.1002/2015JA021318.

Wang, Y. M., P. Z. Ye, S. Wang, and X. H. Xue (2003), An interplanetary cause of large geomagnetic storms: Fast forward shock overtaking preceding magnetic cloud, Geophysical Research Letters, 30(13), doi:10.1029/2002GL016861.

Wilson, L. B., C. A. Cattell, P. J. Kellogg, K. Goetz, K. Kersten, J. C. Kasper, A. Szabo, and K. Meziane (2009), Low-frequency whistler waves and shocklets observed at quasiperpendicular interplanetary shocks, Journal of Geophysical Research: Space Physics, 114(A10), n/a-n/a, doi:10.1029/2009JA014376, a10106.

Wilson, L. B., A. Koval, A. Szabo, A. Breneman, C. A. Cattell, K. Goetz, P. J. Kellogg, K. Kersten, J. C. Kasper, B. A. Maruca, and M. Pulupa (2012), Observations of electromagnetic whistler precursors at supercritical interplanetary shocks, Geophysical Research Letters, 39(8), n/a-n/a, doi:10.1029/2012GL051581, 108109.

Winske, D., and K. B. Quest (1988), Magnetic field and density fluctuations at perpendicular supercritical collisionless shocks, J. Geophys. Res., 93, 9681-9693, doi: 10.1029/JA093iA09p09681.

Yu, W., C. J. Farrugia, A. B. Galvin, N. Lugaz, J. G. Luhmann, K. D. C. Simunac, and E. Kilpua (2016), Small solar wind transients at 1 au: Stereo observations (2007-2014) and comparison with near-earth wind results (1995-2014), J. of Geophys. Res. Space 
Physics, 121(6), 5005-5024, doi:10.1002/2016JA022642.

Zhao, L.-L., G. P. Zank, O. Khabarova, S. Du, Y. Chen, L. Adhikari, and Q. Hu (2018), An unusual energetic particle flux enhancement associated with solar wind magnetic island dynamics, The Astrophysical Journal, 864(2), L34, doi:10.3847/2041-8213/aaddf6.

Zheng, J., and Q. Hu (2018), Observational evidence for self-generation of small-scale magnetic flux ropes from intermittent solar wind turbulence, The Astrophysical Journal Letters, 852(2), L23. 